\title{
Perturbed Companions of Ostrowski's Inequality for Absolutely Continuous Functions (I)
}

Silvestru Sever Dragomir

\begin{abstract}
Perturbed companions of Ostrowski's inequality for absolutely continuous functions whose derivatives are either bounded or of bounded variation and applications are given.
\end{abstract}

AMS Subject Classification (2000). Primary 26D15; Secondary $41 \mathrm{~A} 55$.

Keywords. Ostrowski's inequality, Trapezoid rule, Midpoint rule.

\section{Introduction}

In [16] we established the following companion of Ostrowski inequality [26] for Lebesgue sup-norm:

Theorem 1. Let $f:[a, b] \rightarrow \mathbb{R}$ be an absolutely continuous function on 
$[a, b]$. If $f^{\prime} \in L_{\infty}[a, b]$, then we have the inequalities

$$
\begin{aligned}
& \left|\frac{1}{2}[f(x)+f(a+b-x)]-\frac{1}{b-a} \int_{a}^{b} f(t) d t\right| \\
& \leq \frac{1}{b-a}\left[\frac{(x-a)^{2}}{2}\left\|f^{\prime}\right\|_{[a, x], \infty}+\left(\frac{a+b}{2}-x\right)^{2}\left\|f^{\prime}\right\|_{[x, a+b-x], \infty}\right. \\
& \left.+\frac{(x-a)^{2}}{2}\left\|f^{\prime}\right\|_{[a+b-x, b], \infty}\right] \\
& \leq\left\{\begin{array}{l}
{\left[\frac{1}{8}+2\left(\frac{x-\frac{3 a+b}{4}}{b-a}\right)^{2}\right](b-a)\left\|f^{\prime}\right\|_{[a, b], \infty}} \\
{\left[\frac{1}{2^{\alpha-1}}\left(\frac{x-a}{b-a}\right)^{2 \alpha}+\left(\frac{x-\frac{a+b}{2}}{b-a}\right)^{2 \alpha}\right]^{\frac{1}{\alpha}}} \\
\times\left[\left\|f^{\prime}\right\|_{[a, x], \infty}^{\beta}+\left\|f^{\prime}\right\|_{[x, a+b-x], \infty}^{\beta}+\left\|f^{\prime}\right\|_{[a+b-x, b], \infty}^{\beta}\right]^{\frac{1}{\beta}}(b-a) \\
\max \left\{\frac{1}{2}\left(\frac{x-a}{b-a}\right)^{2},\left(\frac{x-\frac{a+b}{2}}{b-a}\right)^{2}\right\} \\
\times\left[\left\|f^{\prime}\right\|_{[a, x], \infty}+\left\|f^{\prime}\right\|_{[x, a+b-x], \infty}+\left\|f^{\prime}\right\|_{[a+b-x, b], \infty}\right] \\
\end{array}\right.
\end{aligned}
$$

for any $x \in\left[a, \frac{a+b}{2}\right]$, where

$$
\|g\|_{[c, d], \infty}:=e s s \sup _{t \in[c, d]}|g(s)| .
$$

The inequality (1.1), the first inequality in (1.1) and the constant $\frac{1}{8}$ are sharp.

If in Theorem 1 we choose $x=a$, then we get

$$
\left|\frac{f(a)+f(b)}{2}-\frac{1}{b-a} \int_{a}^{b} f(t) d t\right| \leq \frac{1}{4}(b-a)\left\|f^{\prime}\right\|_{[a, b], \infty}
$$

with $\frac{1}{4}$ as a sharp constant (see for example [20, p. 25]). 
If in the same theorem we now choose $x=\frac{a+b}{2}$, then we get

$$
\begin{aligned}
\left|f\left(\frac{a+b}{2}\right)-\frac{1}{b-a} \int_{a}^{b} f(t) d t\right| & \leq \frac{1}{8}(b-a)\left[\left\|f^{\prime}\right\|_{\left[a, \frac{a+b}{2}\right], \infty}+\left\|f^{\prime}\right\|_{\left[\frac{a+b}{2}, b\right], \infty}\right] \\
& \leq \frac{1}{4}(b-a)\left\|f^{\prime}\right\|_{[a, b], \infty}
\end{aligned}
$$

with the constants $\frac{1}{8}$ and $\frac{1}{4}$ being sharp. This result was obtained in [15] by a different argument.

It is natural to consider the following corollary.

Corollary 2. With the assumptions in Theorem 1, one has the inequality:

$$
\left|\frac{1}{2}\left[f\left(\frac{3 a+b}{4}\right)+f\left(\frac{a+3 b}{4}\right)\right]-\frac{1}{b-a} \int_{a}^{b} f(t) d t\right| \leq \frac{1}{8}(b-a)\left\|f^{\prime}\right\|_{[a, b], \infty} .
$$

The constant $\frac{1}{8}$ is best possible in the sense that it cannot be replaced by a smaller quantity.

In the same paper [16] we established the corresponding inequalities for Lebesgue $p$-norms with $p \geq 1$ as well as have provided some applications for cumulative distribution functions and some quadrature rules.

For a monograph devoted to Ostrowski type inequalities, see [20].

For research papers on Ostrowski's inequality see [1]-[19], [21]-[23] and [25].

Motivated by the above results, we investigate in this paper some perturbed versions of the inequality (1.1). Applications for cumulative distribution function are provided as well.

\section{Some Identities}

The following identity holds.

Lemma 3. Assume that $f:[a, b] \rightarrow \mathbb{C}$ is an absolutely continuous function 
on $[a, b]$. Then we have the equality

$$
\begin{aligned}
& \frac{1}{2}[f(x)+f(a+b-x)]+\frac{1}{2}(x-a)^{2} \frac{\lambda_{3}(x)-\lambda_{1}(x)}{b-a}-\frac{1}{b-a} \int_{a}^{b} f(t) d t \\
& =\frac{1}{b-a} \int_{a}^{x}(t-a)\left[f^{\prime}(t)-\lambda_{1}(x)\right] d t \\
& +\frac{1}{b-a} \int_{x}^{a+b-x}\left(t-\frac{a+b}{2}\right)\left[f^{\prime}(t)-\lambda_{2}(x)\right] d t \\
& +\frac{1}{b-a} \int_{a+b-x}^{b}(t-b)\left[f^{\prime}(t)-\lambda_{3}(x)\right] d t,
\end{aligned}
$$

for any $x \in\left[a, \frac{a+b}{2}\right]$ and $\lambda_{j}(x), j=1,2,3$ complex numbers.

Proof. Using the integration by parts formula for Lebesgue integral, we have

$$
\begin{gathered}
\int_{a}^{x}(t-a)\left[f^{\prime}(t)-\lambda_{1}(x)\right] d t \\
=\int_{a}^{x}(t-a) f^{\prime}(t) d t-\lambda_{1}(x) \int_{a}^{x}(t-a) d t \\
=(x-a) f(x)-\int_{a}^{x} f(t) d t-\frac{1}{2} \lambda_{1}(x)(x-a)^{2}, \\
\int_{x}^{a+b-x}\left(t-\frac{a+b}{2}\right)\left[f^{\prime}(t)-\lambda_{2}(x)\right] d t \\
=\int_{x}^{a+b-x}\left(t-\frac{a+b}{2}\right) f^{\prime}(t) d t-\lambda_{2}(x) \int_{x}^{a+b-x}\left(t-\frac{a+b}{2}\right) d t \\
=f(a+b-x)\left(\frac{a+b}{2}-x\right)-f(x)\left(x-\frac{a+b}{2}\right)-\int_{x}^{a+b-x} f(t) d t \\
-\lambda_{2}(x) \int_{x}^{a+b-x}\left(t-\frac{a+b}{2}\right) d t \\
=f(a+b-x)\left(\frac{a+b}{2}-x\right)-f(x)\left(x-\frac{a+b}{2}\right)-\int_{x}^{a+b-x} f(t) d t,
\end{gathered}
$$

since, by symmetry

$$
\int_{x}^{a+b-x}\left(t-\frac{a+b}{2}\right) d t=0
$$


and

$$
\begin{aligned}
& \int_{a+b-x}^{b}(t-b)\left[f^{\prime}(t)-\lambda_{3}(x)\right] d t \\
& =\int_{a+b-x}^{b}(t-b) f^{\prime}(t) d t-\lambda_{3}(x) \int_{a+b-x}^{b}(t-b) d t \\
& =(x-a) f(a+b-x)-\int_{a+b-x}^{b} f(t) d t+\frac{1}{2} \lambda_{3}(x)(x-a)^{2} .
\end{aligned}
$$

Summing the above equalities, we deduce

$$
\begin{aligned}
& \int_{a}^{x}(t-a)\left[f^{\prime}(t)-\lambda_{1}(x)\right] d t+\int_{x}^{a+b-x}\left(t-\frac{a+b}{2}\right)\left[f^{\prime}(t)-\lambda_{2}(x)\right] d t \\
& +\int_{a+b-x}^{b}(t-b)\left[f^{\prime}(t)-\lambda_{3}(x)\right] d t \\
& =(b-a) \frac{f(x)+f(a+b-x)}{2}-\int_{a}^{b} f(t) d t+\frac{1}{2}\left[\lambda_{3}(x)-\lambda_{1}(x)\right](x-a)^{2},
\end{aligned}
$$

which is equivalent with the desired identity (2.1).

The following particular cases are of interest:

Corollary 4. With the assumption of Lemma 3 we have the equalities

$$
\begin{aligned}
& \frac{f(a)+f(b)}{2}-\frac{1}{b-a} \int_{a}^{b} f(t) d t=\frac{1}{b-a} \int_{a}^{b}\left(t-\frac{a+b}{2}\right)\left[f^{\prime}(t)-\lambda_{2}\right] d t \\
& f\left(\frac{a+b}{2}\right)+\frac{1}{8}(b-a)\left(\lambda_{3}-\lambda_{1}\right)-\frac{1}{b-a} \int_{a}^{b} f(t) d t \\
& =\frac{1}{b-a} \int_{a}^{\frac{a+b}{2}}(t-a)\left[f^{\prime}(t)-\lambda_{1}\right] d t+\frac{1}{b-a} \int_{\frac{a+b}{2}}^{b}(t-b)\left[f^{\prime}(t)-\lambda_{3}\right] d t
\end{aligned}
$$


and

$$
\begin{aligned}
& \frac{1}{2}\left[f\left(\frac{3 a+b}{4}\right)+f\left(\frac{a+3 b}{4}\right)\right]+\frac{1}{32}(b-a)\left(\lambda_{3}-\lambda_{1}\right)-\frac{1}{b-a} \int_{a}^{b} f(t) d t \\
& =\frac{1}{b-a} \int_{a}^{\frac{3 a+b}{4}}(t-a)\left[f^{\prime}(t)-\lambda_{1}\right] d t \\
& +\frac{1}{b-a} \int_{\frac{3 a+b}{4}}^{\frac{a+3 b}{4}}\left(t-\frac{a+b}{2}\right)\left[f^{\prime}(t)-\lambda_{2}\right] d t \\
& +\frac{1}{b-a} \int_{\frac{a+3 b}{4}}^{b}(t-b)\left[f^{\prime}(t)-\lambda_{3}\right] d t
\end{aligned}
$$

for any $\lambda_{1}, \lambda_{2}, \lambda_{3} \in \mathbb{C}$.

The following particular result with no parameter in the left hand term holds:

Corollary 5. Assume that $f:[a, b] \rightarrow \mathbb{C}$ is absolutely continuous on $[a, b]$. Then we have the equality

$$
\begin{aligned}
& \frac{1}{2}[f(x)+f(a+b-x)]-\frac{1}{b-a} \int_{a}^{b} f(t) d t \\
& =\frac{1}{b-a} \int_{a}^{x}(t-a)\left[f^{\prime}(t)-\lambda_{1}(x)\right] d t \\
& +\frac{1}{b-a} \int_{x}^{a+b-x}\left(t-\frac{a+b}{2}\right)\left[f^{\prime}(t)-\lambda_{2}(x)\right] d t \\
& +\frac{1}{b-a} \int_{a+b-x}^{b}(t-b)\left[f^{\prime}(t)-\lambda_{1}(x)\right] d t,
\end{aligned}
$$

for any $x \in\left[a, \frac{a+b}{2}\right]$ and $\lambda_{i}(x), i=1,2$ complex numbers.

Remark 1. We get from (2.3) the following particular case:

$$
\begin{aligned}
& f\left(\frac{a+b}{2}\right)-\frac{1}{b-a} \int_{a}^{b} f(t) d t \\
& =\frac{1}{b-a} \int_{a}^{\frac{a+b}{2}}(t-a)\left[f^{\prime}(t)-\lambda_{1}\right] d t+\frac{1}{b-a} \int_{\frac{a+b}{2}}^{b}(t-b)\left[f^{\prime}(t)-\lambda_{1}\right] d t
\end{aligned}
$$


for any $\lambda_{1} \in \mathbb{C}$, while from (2.4) we get

$$
\begin{aligned}
& \frac{1}{2}\left[f\left(\frac{3 a+b}{4}\right)+f\left(\frac{a+3 b}{4}\right)\right]-\frac{1}{b-a} \int_{a}^{b} f(t) d t \\
& =\frac{1}{b-a} \int_{a}^{\frac{3 a+b}{4}}(t-a)\left[f^{\prime}(t)-\lambda_{1}\right] d t \\
& +\frac{1}{b-a} \int_{\frac{3 a+b}{4}}^{\frac{a+3 b}{4}}\left(t-\frac{a+b}{2}\right)\left[f^{\prime}(t)-\lambda_{2}\right] d t \\
& +\frac{1}{b-a} \int_{\frac{a+3 b}{4}}^{b}(t-b)\left[f^{\prime}(t)-\lambda_{1}\right] d t
\end{aligned}
$$

for any $\lambda_{1}, \lambda_{2} \in \mathbb{C}$.

\section{Inequalities for Bounded Derivatives}

Now, for $\gamma, \Gamma \in \mathbb{C}$ and $[a, b]$ an interval of real numbers, define the sets of complex-valued functions

$\bar{U}_{[a, b]}(\gamma, \Gamma)$

$:=\{f:[a, b] \rightarrow \mathbb{C} \mid \operatorname{Re}[(\Gamma-f(t))(\overline{f(t)}-\bar{\gamma})] \geq 0$ for almost every $t \in[a, b]\}$

and

$\bar{\Delta}_{[a, b]}(\gamma, \Gamma):=\left\{f:[a, b] \rightarrow \mathbb{C}|| f(t)-\frac{\gamma+\Gamma}{2}\left|\leq \frac{1}{2}\right| \Gamma-\gamma \mid\right.$ for a.e. $\left.t \in[a, b]\right\}$.

The following representation result may be stated.

Proposition 6. For any $\gamma, \Gamma \in \mathbb{C}, \gamma \neq \Gamma$, we have that $\bar{U}_{[a, b]}(\gamma, \Gamma)$ and $\bar{\Delta}_{[a, b]}(\gamma, \Gamma)$ are nonempty, convex and closed sets and

$$
\bar{U}_{[a, b]}(\gamma, \Gamma)=\bar{\Delta}_{[a, b]}(\gamma, \Gamma) .
$$

Proof. We observe that for any $z \in \mathbb{C}$ we have the equivalence

$$
\left|z-\frac{\gamma+\Gamma}{2}\right| \leq \frac{1}{2}|\Gamma-\gamma|
$$

if and only if

$$
\operatorname{Re}[(\Gamma-z)(\bar{z}-\bar{\gamma})] \geq 0
$$


This follows by the equality

$$
\frac{1}{4}|\Gamma-\gamma|^{2}-\left|z-\frac{\gamma+\Gamma}{2}\right|^{2}=\operatorname{Re}[(\Gamma-z)(\bar{z}-\bar{\gamma})]
$$

that holds for any $z \in \mathbb{C}$.

The equality (3.1) is thus a simple consequence of this fact. that:

On making use of the complex numbers field properties we can also state

Corollary 7. For any $\gamma, \Gamma \in \mathbb{C}, \gamma \neq \Gamma$, we have that

$$
\begin{aligned}
\bar{U}_{[a, b]}(\gamma, \Gamma)= & \{f:[a, b] \rightarrow \mathbb{C} \mid(\operatorname{Re} \Gamma-\operatorname{Re} f(t))(\operatorname{Re} f(t)-\operatorname{Re} \gamma) \\
& +(\operatorname{Im} \Gamma-\operatorname{Im} f(t))(\operatorname{Im} f(t)-\operatorname{Im} \gamma) \geq 0 \text { for a.e. } t \in[a, b]\} .
\end{aligned}
$$

Now, if we assume that $\operatorname{Re}(\Gamma) \geq \operatorname{Re}(\gamma)$ and $\operatorname{Im}(\Gamma) \geq \operatorname{Im}(\gamma)$, then we can define the following set of functions as well:

$$
\begin{array}{r}
\bar{S}_{[a, b]}(\gamma, \Gamma):=\{f:[a, b] \rightarrow \mathbb{C} \mid \operatorname{Re}(\Gamma) \geq \operatorname{Re} f(t) \geq \operatorname{Re}(\gamma) \\
\text { and } \operatorname{Im}(\Gamma) \geq \operatorname{Im} f(t) \geq \operatorname{Im}(\gamma) \text { for a.e. } t \in[a, b]\} .
\end{array}
$$

One can easily observe that $\bar{S}_{[a, b]}(\gamma, \Gamma)$ is closed, convex and

$$
\emptyset \neq \bar{S}_{[a, b]}(\gamma, \Gamma) \subseteq \bar{U}_{[a, b]}(\gamma, \Gamma) .
$$

Theorem 8. Assume that $f:[a, b] \rightarrow \mathbb{C}$ is an absolutely continuous function on $[a, b]$ and $x \in\left[a, \frac{a+b}{2}\right]$. If there exist the complex numbers $\gamma_{j}(x) \neq \Gamma_{j}(x)$, $j=1,2,3$ such that

$$
\begin{aligned}
f^{\prime} & \in \bar{\Delta}_{[a, x]}\left(\gamma_{1}(x), \Gamma_{1}(x)\right) \cap \bar{\Delta}_{[x, a+b-x]}\left(\gamma_{2}(x), \Gamma_{2}(x)\right) \\
& \cap \bar{\Delta}_{[a+b-x, b]}\left(\gamma_{3}(x), \Gamma_{3}(x)\right),
\end{aligned}
$$

then we have the inequality

$$
\begin{aligned}
& \mid \frac{1}{2}[f(x)+f(a+b-x)]+\frac{1}{4}(x-a)^{2} \frac{\gamma_{3}(x)+\Gamma_{3}(x)-\gamma_{1}(x)-\Gamma_{1}(x)}{b-a} \\
& -\frac{1}{b-a} \int_{a}^{b} f(t) d t \mid \\
& \leq \frac{1}{4(b-a)}\left[\left|\Gamma_{1}(x)-\gamma_{1}(x)\right|(x-a)^{2}+2\left|\Gamma_{2}(x)-\gamma_{2}(x)\right|\left(\frac{a+b}{2}-x\right)^{2}\right. \\
& \left.+\left|\Gamma_{3}(x)-\gamma_{3}(x)\right|(x-a)^{2}\right] .
\end{aligned}
$$


Proof. Taking the modulus in the equality (2.1) written for

$$
\begin{aligned}
& \lambda_{1}(x)=\frac{\gamma_{1}(x)+\Gamma_{1}(x)}{2}, \lambda_{2}(x)=\frac{\gamma_{2}(x)+\Gamma_{2}(x)}{2} \\
& \lambda_{3}(x)=\frac{\gamma_{3}(x)+\Gamma_{3}(x)}{2}
\end{aligned}
$$

and utilizing the condition (3.5) we have

$$
\begin{aligned}
& \mid \frac{1}{2}[f(x)+f(a+b-x)]+\frac{1}{4}(x-a)^{2} \frac{\gamma_{3}(x)+\Gamma_{3}(x)-\gamma_{1}(x)-\Gamma_{1}(x)}{b-a} \\
& -\frac{1}{b-a} \int_{a}^{b} f(t) d t \mid \\
& \leq \frac{1}{b-a} \int_{a}^{x}(t-a)\left|f^{\prime}(t)-\frac{\gamma_{1}(x)+\Gamma_{1}(x)}{2}\right| d t \\
& +\frac{1}{b-a} \int_{x}^{a+b-x}\left|t-\frac{a+b}{2}\right|\left|f^{\prime}(t)-\frac{\gamma_{2}(x)+\Gamma_{2}(x)}{2}\right| d t \\
& +\frac{1}{b-a} \int_{a+b-x}^{b}(b-t)\left|f^{\prime}(t)-\frac{\gamma_{3}(x)+\Gamma_{3}(x)}{2}\right| d t \\
& \leq \frac{1}{4(b-a)}\left|\Gamma_{1}(x)-\gamma_{1}(x)\right|(x-a)^{2} \\
& +\frac{2}{4(b-a)}\left|\Gamma_{2}(x)-\gamma_{2}(x)\right|\left(\frac{a+b}{2}-x\right)^{2} \\
& +\frac{1}{4(b-a)}\left|\Gamma_{3}(x)-\gamma_{3}(x)\right|(x-a)^{2}
\end{aligned}
$$

and the inequality (3.6) is proved.

Corollary 9. Assume that $f:[a, b] \rightarrow \mathbb{C}$ is an absolutely continuous function on $[a, b]$ and $x \in\left[a, \frac{a+b}{2}\right]$. If there exist the complex numbers $\gamma_{j}(x) \neq \Gamma_{j}(x)$, $j=1,2$ such that

$$
\begin{aligned}
f^{\prime} & \in \bar{\Delta}_{[a, x]}\left(\gamma_{1}(x), \Gamma_{1}(x)\right) \cap \bar{\Delta}_{[x, a+b-x]}\left(\gamma_{2}(x), \Gamma_{2}(x)\right) \\
& \cap \bar{\Delta}_{[a+b-x, b]}\left(\gamma_{1}(x), \Gamma_{1}(x)\right),
\end{aligned}
$$

then we have the inequality

$$
\begin{aligned}
& \left|\frac{1}{2}[f(x)+f(a+b-x)]-\frac{1}{b-a} \int_{a}^{b} f(t) d t\right| \\
& \leq \frac{1}{2(b-a)}\left[\left|\Gamma_{1}(x)-\gamma_{1}(x)\right|(x-a)^{2}+\left|\Gamma_{2}(x)-\gamma_{2}(x)\right|\left(\frac{a+b}{2}-x\right)^{2}\right] .
\end{aligned}
$$


Remark 2. Assume that $f:[a, b] \rightarrow \mathbb{C}$ is an absolutely continuous function on $[a, b]$.

If there exist the complex numbers $\gamma_{2} \neq \Gamma_{2}$ such that $f^{\prime} \in \bar{\Delta}_{[a, b]}\left(\gamma_{2}, \Gamma_{2}\right)$, then

$$
\left|\frac{1}{2}[f(a)+f(b)]-\frac{1}{b-a} \int_{a}^{b} f(t) d t\right| \leq \frac{1}{8}(b-a)\left|\Gamma_{2}-\gamma_{2}\right| .
$$

If there exist the complex numbers $\gamma_{j} \neq \Gamma_{j}, j=1,3$ such that

$$
f^{\prime} \in \bar{\Delta}_{\left[a, \frac{a+b}{2}\right]}\left(\gamma_{1}, \Gamma_{1}\right) \cap \bar{\Delta}_{\left[\frac{a+b}{2}, b\right]}\left(\gamma_{3}, \Gamma_{3}\right) \text {, }
$$

then we have the inequality

$$
\begin{aligned}
& \left|f\left(\frac{a+b}{2}\right)+\frac{1}{16}(b-a)\left(\Gamma_{3}+\gamma_{3}-\Gamma_{1}-\gamma_{1}\right)-\frac{1}{b-a} \int_{a}^{b} f(t) d t\right| \\
& \leq \frac{1}{16}(b-a)\left[\left|\Gamma_{1}-\gamma_{1}\right|+\left|\Gamma_{3}-\gamma_{3}\right|\right] .
\end{aligned}
$$

In particular, if $f^{\prime} \in \bar{\Delta}_{[a, b]}\left(\gamma_{1}, \Gamma_{1}\right)$ then

$$
\left|f\left(\frac{a+b}{2}\right)-\frac{1}{b-a} \int_{a}^{b} f(t) d t\right| \leq \frac{1}{8}(b-a)\left|\Gamma_{1}-\gamma_{1}\right| .
$$

If there exist the complex numbers $\gamma_{j} \neq \Gamma_{j}, j=1,2,3$ such that

$$
f^{\prime} \in \bar{\Delta}_{\left[a, \frac{3 a+b}{4}\right]}\left(\gamma_{1}, \Gamma_{1}\right) \cap \bar{\Delta}_{\left[\frac{3 a+b}{4}, \frac{a+3 b}{4}\right]}\left(\gamma_{2}, \Gamma_{2}\right) \cap \bar{\Delta}_{\left[\frac{a+3 b}{4}, b\right]}\left(\gamma_{3}, \Gamma_{3}\right),
$$

then

$$
\begin{aligned}
& \mid \frac{1}{2}\left[f\left(\frac{3 a+b}{4}\right)+f\left(\frac{a+3 b}{4}\right)\right]+\frac{1}{64}(b-a)\left(\Gamma_{3}+\gamma_{3}-\Gamma_{1}-\gamma_{1}\right) \\
& -\frac{1}{b-a} \int_{a}^{b} f(t) d t \mid \\
& \leq \frac{1}{64}(b-a)\left[\left|\Gamma_{1}-\gamma_{1}\right|+2\left|\Gamma_{2}-\gamma_{2}\right|+\left|\Gamma_{3}-\gamma_{3}\right|\right] .
\end{aligned}
$$

In particular, if $\gamma_{3}=\gamma_{1}$ and $\Gamma_{3}=\Gamma_{1}$, then

$$
\begin{aligned}
& \left|\frac{1}{2}\left[f\left(\frac{3 a+b}{4}\right)+f\left(\frac{a+3 b}{4}\right)\right]-\frac{1}{b-a} \int_{a}^{b} f(t) d t\right| \\
& \leq \frac{1}{32}(b-a)\left[\left|\Gamma_{1}-\gamma_{1}\right|+\left|\Gamma_{2}-\gamma_{2}\right|\right],
\end{aligned}
$$


provided

$$
f^{\prime} \in \bar{\Delta}_{\left[a, \frac{3 a+b}{4}\right]}\left(\gamma_{1}, \Gamma_{1}\right) \cap \bar{\Delta}_{\left[\frac{3 a+b}{4}, \frac{a+3 b}{4}\right]}\left(\gamma_{2}, \Gamma_{2}\right) \cap \bar{\Delta}_{\left[\frac{a+3 b}{4}, b\right]}\left(\gamma_{1}, \Gamma_{1}\right) .
$$

Moreover, if $f^{\prime} \in \bar{\Delta}_{[a, b]}\left(\gamma_{1}, \Gamma_{1}\right)$ then

$$
\begin{aligned}
& \left|\frac{1}{2}\left[f\left(\frac{3 a+b}{4}\right)+f\left(\frac{a+3 b}{4}\right)\right]-\frac{1}{b-a} \int_{a}^{b} f(t) d t\right| \\
& \leq \frac{1}{16}(b-a)\left|\Gamma_{1}-\gamma_{1}\right| .
\end{aligned}
$$

The case of real-valued functions is of interest.

Remark 3. If the function $f:[a, b] \rightarrow \mathbb{R}$ is absolutely continuous and if there exist the constants $l<L$ such that $l \leq f^{\prime}(t) \leq L$ for almost every $t \in[a, b]$, then we have the inequalities

$$
\begin{array}{r}
\left|\frac{1}{2}[f(a)+f(b)]-\frac{1}{b-a} \int_{a}^{b} f(t) d t\right| \leq \frac{1}{8}(b-a)(L-l), \\
\left|f\left(\frac{a+b}{2}\right)-\frac{1}{b-a} \int_{a}^{b} f(t) d t\right| \leq \frac{1}{8}(b-a)(L-l)
\end{array}
$$

and

$$
\begin{aligned}
& \left|\frac{1}{2}\left[f\left(\frac{3 a+b}{4}\right)+f\left(\frac{a+3 b}{4}\right)\right]-\frac{1}{b-a} \int_{a}^{b} f(t) d t\right| \\
& \leq \frac{1}{16}(b-a)(L-l) .
\end{aligned}
$$

These results improve the corresponding inequalities from Introduction.

\section{Inequalities for Derivatives of Bounded Variation}

Assume that $f: I \rightarrow \mathbb{C}$ is an absolutely continuous function on $[a, b] \subset \stackrel{\circ}{I}$, the interior of $I$. Then from (2.1) we have for $\lambda_{1}(x)=f^{\prime}(a), \lambda_{2}(x)=$ 
$\frac{f^{\prime}(x)+f^{\prime}(a+b-x)}{2}$ and $\lambda_{3}(x)=f^{\prime}(b)$ the equality

$$
\begin{aligned}
& \frac{1}{2}[f(x)+f(a+b-x)]+\frac{1}{2}(x-a)^{2} \frac{f^{\prime}(b)-f^{\prime}(a)}{b-a}-\frac{1}{b-a} \int_{a}^{b} f(t) d t \\
& =\frac{1}{b-a} \int_{a}^{x}(t-a)\left[f^{\prime}(t)-f^{\prime}(a)\right] d t \\
& +\frac{1}{b-a} \int_{x}^{a+b-x}\left(t-\frac{a+b}{2}\right)\left[f^{\prime}(t)-\frac{f^{\prime}(x)+f^{\prime}(a+b-x)}{2}\right] d t \\
& +\frac{1}{b-a} \int_{a+b-x}^{b}(t-b)\left[f^{\prime}(t)-f^{\prime}(b)\right] d t
\end{aligned}
$$

for any $x \in\left[a, \frac{a+b}{2}\right]$.

We can state the following result.

Theorem 10. Assume that $f: I \rightarrow \mathbb{C}$ is an absolutely continuous function on $[a, b] \subset \stackrel{\circ}{I}$. If the derivative $f^{\prime}$ is of bounded variation on $[a, b]$, then

$$
\begin{aligned}
& \left|\frac{1}{2}[f(x)+f(a+b-x)]+\frac{1}{2}(x-a)^{2} \frac{f^{\prime}(b)-f^{\prime}(a)}{b-a}-\frac{1}{b-a} \int_{a}^{b} f(t) d t\right| \\
& \leq \frac{1}{b-a} \int_{a}^{x}(t-a) \bigvee_{a}^{t}\left(f^{\prime}\right) d t+\frac{1}{2(b-a)}\left(x-\frac{a+b}{2}\right) \bigvee_{x}^{2 a+b-x}\left(f^{\prime}\right) \\
& +\frac{1}{b-a} \int_{a+b-x}^{b}(b-t) \bigvee_{t}^{b}\left(f^{\prime}\right) d t \\
& \leq \frac{1}{2(b-a)}\left[(x-a)^{2} \bigvee_{a}^{x}\left(f^{\prime}\right)+\left(x-\frac{a+b}{2}\right)^{2} \bigvee_{x}^{a+b-x}\left(f^{\prime}\right)+(x-a)^{2} \bigvee_{a+b-x}^{b}\left(f^{\prime}\right)\right] \\
& \leq\left\{\begin{array}{c}
\frac{1}{2(b-a)} \max \left\{(x-a)^{2},\left(x-\frac{a+b}{2}\right)^{2}\right\} \bigvee_{a}^{b}\left(f^{\prime}\right) \\
\frac{1}{2(b-a)} \max \left\{\bigvee_{a}^{x}\left(f^{\prime}\right), \bigvee_{x}^{a+b-x}\left(f^{\prime}\right), \bigvee_{a+b-x}^{b}\left(f^{\prime}\right)\right\}\left[2(x-a)^{2}+\left(x-\frac{a+b}{2}\right)^{2}\right]
\end{array}\right.
\end{aligned}
$$

for any $x \in\left[a, \frac{a+b}{2}\right]$. 
Proof. If we take the modulus in (4.1) we get

$$
\begin{aligned}
& \left|\frac{1}{2}[f(x)+f(a+b-x)]+\frac{1}{2}(x-a)^{2} \frac{f^{\prime}(b)-f^{\prime}(a)}{b-a}-\frac{1}{b-a} \int_{a}^{b} f(t) d t\right| \\
& \leq \frac{1}{b-a} \int_{a}^{x}(t-a)\left|f^{\prime}(t)-f^{\prime}(a)\right| d t \\
& +\frac{1}{b-a} \int_{x}^{a+b-x}\left|t-\frac{a+b}{2}\right|\left|f^{\prime}(t)-\frac{f^{\prime}(x)+f^{\prime}(a+b-x)}{2}\right| d t \\
& +\frac{1}{b-a} \int_{a+b-x}^{b}(b-t)\left|f^{\prime}(t)-f^{\prime}(b)\right| d t:=K
\end{aligned}
$$

for any $x \in\left[a, \frac{a+b}{2}\right]$.

Let $x \in\left(a, \frac{a+b}{2}\right)$. Since $f^{\prime}$ is of bounded variation on $[a, b]$, then

$$
\left|f^{\prime}(t)-f^{\prime}(a)\right| \leq \bigvee_{a}^{t}\left(f^{\prime}\right)
$$

for any $t \in[a, x]$ and

$$
\begin{aligned}
& \left|f^{\prime}(t)-\frac{f^{\prime}(x)+f^{\prime}(a+b-x)}{2}\right| \\
& =\left|\frac{f^{\prime}(t)-f^{\prime}(x)+f^{\prime}(t)-f^{\prime}(a+b-x)}{2}\right| \\
& \leq \frac{1}{2}\left[\left|f^{\prime}(t)-f^{\prime}(x)\right|+\left|f^{\prime}(a+b-x)-f^{\prime}(t)\right|\right] \leq \frac{1}{2} \bigvee_{x}^{a+b-x}\left(f^{\prime}\right)
\end{aligned}
$$

for any $t \in[x, a+b-x]$.

We also have

$$
\left|f^{\prime}(t)-f^{\prime}(b)\right| \leq \bigvee_{t}^{b}\left(f^{\prime}\right), t \in[a+b-x, b]
$$


Then we get

$$
\begin{aligned}
K & \leq \frac{1}{b-a} \int_{a}^{x}(t-a) \bigvee_{a}^{t}\left(f^{\prime}\right) d t+\frac{1}{2(b-a)} \bigvee_{x}^{a+b-x}\left(f^{\prime}\right) \int_{x}^{a+b-x}\left|t-\frac{a+b}{2}\right| d t \\
& +\frac{1}{b-a} \int_{a+b-x}^{b}(b-t) \bigvee_{t}^{b}\left(f^{\prime}\right) d t \\
& \leq \frac{1}{b-a} \bigvee_{a}^{x}\left(f^{\prime}\right) \int_{a}^{x}(t-a) d t+\frac{1}{2(b-a)} \bigvee_{x}^{a+b-x}\left(f^{\prime}\right) \int_{x}^{a+b-x}\left|t-\frac{a+b}{2}\right| d t \\
+ & \frac{1}{b-a} \bigvee_{a+b-x}^{b}\left(f^{\prime}\right) \int_{a+b-x}^{b}(b-t) d t \\
& =\frac{1}{2(b-a)}(x-a)^{2} \bigvee_{a}^{x}\left(f^{\prime}\right)+\frac{1}{2(b-a)}\left(x-\frac{a+b}{2}\right)^{2 a+b-x} \bigvee_{x}\left(f^{\prime}\right) \\
& +\frac{1}{2(b-a)}(x-a)^{2} \bigvee_{a+b-x}^{b}\left(f^{\prime}\right),
\end{aligned}
$$

which proves the first two inequalities in (4.2).

The last part is obvious by the maximum properties.

Corollary 11. With the assumptions of Theorem 10 we have

$$
\begin{aligned}
& \left|\frac{1}{2}[f(a)+f(b)]-\frac{1}{b-a} \int_{a}^{b} f(t) d t\right| \leq \frac{1}{8}(b-a) \bigvee_{a}^{b}\left(f^{\prime}\right), \\
& \left|f\left(\frac{a+b}{2}\right)+\frac{1}{8}(b-a)\left[f^{\prime}(b)-f^{\prime}(a)\right]-\frac{1}{b-a} \int_{a}^{b} f(t) d t\right| \\
& \leq \frac{1}{b-a} \int_{a}^{\frac{a+b}{2}}(t-a) \bigvee_{a}^{t}\left(f^{\prime}\right) d t+\frac{1}{b-a} \int_{\frac{a+b}{2}}^{b}(b-t) \bigvee_{t}^{b}\left(f^{\prime}\right) d t \\
& \leq \frac{1}{8}(b-a) \bigvee_{a}^{b}\left(f^{\prime}\right)
\end{aligned}
$$


and

$$
\begin{aligned}
& \mid \frac{1}{2}\left[f\left(\frac{3 a+b}{4}\right)+f\left(\frac{a+3 b}{4}\right)\right]+\frac{1}{32}(b-a)\left[f^{\prime}(b)-f^{\prime}(a)\right] \\
& -\frac{1}{b-a} \int_{a}^{b} f(t) d t \mid \\
& \leq \frac{1}{b-a} \int_{a}^{\frac{3 a+b}{4}}(t-a) \bigvee_{a}^{t}\left(f^{\prime}\right) d t+\frac{1}{32}(b-a) \bigvee_{\frac{3 a+b}{4}}^{\frac{a+3 b}{4}}\left(f^{\prime}\right) \\
& +\frac{1}{b-a} \int_{\frac{a+3 b}{4}}^{b}(b-t) \bigvee_{t}^{b}\left(f^{\prime}\right) d t \\
& \leq \frac{1}{32}(b-a) \bigvee_{a}^{b}\left(f^{\prime}\right) .
\end{aligned}
$$

\section{Applications for PDF}

Now, let $X$ be a random variable taking values in the finite interval $[a, b]$, with the probability density function (PDF) $f:[a, b] \rightarrow[0, \infty)$ and with the cumulative distribution function (CDF) $F(x)=\operatorname{Pr}(X \leq x)=\int_{a}^{x} f(t) d t$. We know that $F$ is monotonic nondecreasing and absolutely continuous on $[a, b], F^{\prime}=f$ almost everywhere on $[a, b]$ and $F(a)=0, F(b)=\int_{a}^{b} f(t) d t=$ 1 .

Assume that $g:[a, b] \rightarrow \mathbb{R}$ is an absolutely continuous function on $[a, b]$ and there exist the constants $m<M$ such that

$$
m \leq g^{\prime}(t) \leq M \text { for almost every } t \in[a, b]
$$

then, by Corollary 9, we have the inequality

$$
\begin{aligned}
& \left|\frac{1}{2}[g(x)+g(a+b-x)]-\frac{1}{b-a} \int_{a}^{b} g(t) d t\right| \\
& \leq \frac{1}{2}(M-m)\left[\frac{1}{8}+2\left(\frac{x-\frac{3 a+b}{4}}{b-a}\right)^{2}\right](b-a)
\end{aligned}
$$

for any $x \in\left[a, \frac{a+b}{2}\right]$. 
Proposition 12. Let $X$ be a random variable taking values in the finite interval $[a, b]$, with PDF $f:[a, b] \rightarrow[0, \infty)$ and with $C D F F(x)=\operatorname{Pr}(X \leq x)=$ $\int_{a}^{x} f(t) d t$. If there exist the constants $m<M$ such that

$$
m \leq f(t) \leq M \text { for almost every } t \in[a, b]
$$

then,

$$
\begin{aligned}
& \left|\frac{1}{2}[F(x)+F(a+b-x)]-\frac{b-E(X)}{b-a}\right| \\
& \leq \frac{1}{2}(M-m)\left[\frac{1}{8}+2\left(\frac{x-\frac{3 a+b}{4}}{b-a}\right)^{2}\right](b-a)
\end{aligned}
$$

for any $x \in\left[a, \frac{a+b}{2}\right]$, where $E(X)=\int_{a}^{b} t d F(t)$ is the expectation of $X$.

Proof. Follows from (5.1) for $g=F$ and by taking into account that

$$
\int_{a}^{b} F(t) d t=b-E(X)
$$

Corollary 13. With the assumptions in Proposition 12, we have

$$
\left|\frac{1}{2}\left[F\left(\frac{3 a+b}{4}\right)+F\left(\frac{a+3 b}{4}\right)\right]-\frac{b-E(X)}{b-a}\right| \leq \frac{1}{16}(M-m)(b-a)
$$

Utilising Theorem 10 we can also state:

Proposition 14. If PDF $f:[a, b] \rightarrow[0, \infty)$ is of bounded variation on $[a, b]$, 
then

$$
\begin{aligned}
& \left|\frac{1}{2}[F(x)+F(a+b-x)]+\frac{1}{2}(x-a)^{2} \frac{f(b)-f(a)}{b-a}-\frac{b-E(X)}{b-a}\right| \\
& \leq \frac{1}{b-a} \int_{a}^{x}(t-a) \bigvee_{a}^{t}(f) d t+\frac{1}{2(b-a)}\left(x-\frac{a+b}{2}\right) \bigvee_{x}^{2 a+b-x}(f) \\
& +\frac{1}{b-a} \int_{a+b-x}^{b}(b-t) \bigvee_{t}^{b}(f) d t \\
& \leq \frac{1}{2(b-a)} \\
& \times\left[(x-a)^{2} \bigvee_{a}^{x}(f)+\left(x-\frac{a+b}{2}\right)^{2} \bigvee_{x}^{a+b-x}(f)+(x-a)^{2} \bigvee_{a+b-x}^{b}(f)\right] \\
& \leq \frac{1}{2(b-a)} \\
& \times\left\{\begin{array}{l}
\max \left\{(x-a)^{2},\left(x-\frac{a+b}{2}\right)^{2}\right\} \bigvee_{a}^{b}(f), \\
\max \left\{\bigvee_{a}^{x}(f), \bigvee_{x}^{a+b-x}(f), \bigvee_{a+b-x}^{b}(f)\right\}\left[2(x-a)^{2}+\left(x-\frac{a+b}{2}\right)^{2}\right]
\end{array}\right.
\end{aligned}
$$

for any $x \in\left[a, \frac{a+b}{2}\right]$.

Finally we have: 
Corollary 15. With the assumptions in Proposition 14, we have

$$
\begin{aligned}
& \mid \frac{1}{2}\left[F\left(\frac{3 a+b}{4}\right)+F\left(\frac{a+3 b}{4}\right)\right]+\frac{1}{32}(b-a)[f(b)-f(a)] \\
& -\frac{b-E(X)}{b-a} \mid \\
& \leq \frac{1}{b-a} \int_{a}^{\frac{3 a+b}{4}}(t-a) \bigvee_{a}^{t}(f) d t+\frac{1}{32}(b-a) \bigvee_{\frac{3 a+b}{4}}^{\frac{a+3 b}{4}}(f) \\
& +\frac{1}{b-a} \int_{\frac{a+3 b}{4}}^{b}(b-t) \bigvee_{t}^{b}(f) d t \\
& \leq \frac{1}{32}(b-a) \bigvee_{a}^{b}(f) .
\end{aligned}
$$

\section{References}

[1] G. A. Anastassiou, Univariate Ostrowski inequalities, revisited, Monatsh. Math., (2002), 175-189.

[2] P. Cerone and S. S. Dragomir, Midpoint-type rules from an inequalities point of view, Ed. G. A. Anastassiou, Handbook of Analytic-Computational Methods in Applied Mathematics, CRC Press, New York, 135-200, 2000.

[3] P. Cerone and S. S. Dragomir, New bounds for the three-point rule involving the Riemann-Stieltjes integrals, Advances in Statistics Combinatorics and Related Areas, C. Gulati, et al., World Science Publishing, 53-62, 2002.

[4] P. Cerone, S. S. Dragomir, and C. E. M. Pearce, A generalised trapezoid inequality for functions of bounded variation, Turkish J. Math., (2000), 147-163.

[5] P. Cerone, S. S. Dragomir, and J. Roumeliotis, Some Ostrowski type inequalities for time differentiable mappings and applications, Demonstratio Mathematica, (1999), 697-712.

[6] S. S. Dragomir, Ostrowski's inequality for monotonous mappings and applications, J. Korean Soc. Ind. Appl. Math., (1999), 127-135.

[7] S. S. Dragomir, The Ostrowski's integral inequality for Lipschitzian mappings and applications, Comp. Math. Appl., (1999), 33-37.

[8] S. S. Dragomir, The Ostrowski integral inequality for mappings of bounded variation, Bull. Austral. Math. Soc., (1999), 495-508.

[9] S. S. Dragomir, On the midpoint quadrature formula for mappings with bounded variation and applications, Kragujevac J. Math., (2000), 13-18. 
[10] S. S. Dragomir, On the Ostrowski's inequality for Riemann-Stieltjes integral, Korean J. Appl. Math., (2000), 477-485.

[11] S. S. Dragomir, On the Ostrowski's integral inequality for mappings with bounded variation and applications, Math. Ineq. Appl., (2001), 33-40.

[12] S. S. Dragomir, On the Ostrowski inequality for Riemann-Stieltjes integral where is of Holder type and is of bounded variation and applications, J. Korean Soc. Ind. Appl. Math., (2001), 35-45.

[13] S. S. Dragomir, Ostrowski type inequalities for isotonic linear functionals, J. Inequal. Pure Appl. Math., Art. 68., (2002)

[14] S. S. Dragomir, An inequality improving the first Hermite-Hadamard inequality for convex functions defined on linear spaces and applications for semi-inner products, $J$. Inequal. Pure Appl. Math., no. 2 (Article 31), (2002), 8 pp.

[15] S. S. Dragomir, A refinement of Ostrowski's inequality for absolutely continuous functions whose derivatives belong to and applications, Libertas Math., (2002), 49-63.

[16] S. S. Dragomir, Some companions of Ostrowski's inequality for absolutely continuous functions and applications. Preprint RGMIA Res. Rep. Coll., Bull. Korean Math. Soc., no. 2 (Suppl. Art. 29.), (2005), 213-230.

[17] S. S. Dragomir, An Ostrowski like inequality for convex functions and applications, Revista Math. Complutense, (2003), 373-382.

[18] S. S. Dragomir, Operator Inequalities of Ostrowski and Trapezoidal Type, Springer Briefs in Mathematics, Springer, New York, 2012

[19] S. S. Dragomir, P. Cerone, J. Roumeliotis, and S. Wang, A weighted version of Ostrowski inequality for mappings of Holder type and applications in numerical analysis, Bull. Math. Soc. Sci. Math., (1999), 301-314.

[20] S. S. Dragomir and Th. M. Rassias, Ostrowski Type Inequalities and Applications in Numerical Integration, Kluwer Academic Publishers, Dordrecht/Boston/London, 2002 .

[21] S. S. Dragomir and S. Wang, A new inequality of Ostrowski's type in norm and applications to some special means and to some numerical quadrature rules, Tamkang J. of Math., (1997), 239-244.

[22] S. S. Dragomir and S. Wang, Applications of Ostrowski's inequality to the estimation of error bounds for some special means and some numerical quadrature rules, Appl. Math. Lett., (1998), 105-109.

[23] S. S. Dragomir and S. Wang, A new inequality of Ostrowski's type in norm and applications to some special means and to some numerical quadrature rules, Indian J. of Math., (1998), 245-304.

[24] A. Guessab and G. Schmeisser, Sharp integral inequalities of the HermiteHadamard type, J. Approx. Th., (2002), 260-288.

[25] A. M. Fink, Bounds on the deviation of a function from its averages, Czechoslovak Math. J., No. 2, (1992), 298-310.

[26] A. Ostrowski, Uber die Absolutabweichung einer differentienbaren Funktionen von ihren Integralmittelwert, Comment. Math. Hel., (1938), 226-227. 
Silvestru Sever Dragomir

Mathematics, College of Engineering \& Science

Victoria University, PO Box 14428

Melbourne City, MC 8001, Australia

and

DST-NRF Centre of Excellence in the Mathematical and Statistical Sciences

School of Computer Science and Applied Mathematics

University of the Witwatersrand

Private Bag 3, Johannesburg 2050, South Africa

E-mail: sever.dragomir@vu.edu .au

Received: 18.05.2016

Accepted: 2.06.2016 